\title{
Toxoplasmosis as a cause for behaviour disorders - overview of evidence and mechanisms
}

\author{
Abebaw Fekadu ${ }^{1,2}$, Teshome Shibre ${ }^{1}$ and Anthony J. Cleare ${ }^{2}$ \\ ${ }^{1}$ Department of Psychiatry, Faculty of Medicine, Addis Ababa University, Addis Ababa, Ethiopia; \\ ${ }^{2}$ King's College London, Institute of Psychiatry, Department of Psychological Medicine and Psychiatry, London, UK
}

\begin{abstract}
Background - There is growing interest in the role of microbial agents in the causation of psychiatric disorders. The neurotropic protozoan parasite Toxoplasma gondii is one of the main candidates and has been associated with various psychiatric conditions, including schizophrenia. Methods - A narrative review of the literature from the main medical databases (Medline, PubMed, PsycINFO), Google Scholar and Google using combinations of applicable terms. Results - T. gondii affects the brain in both the acute and the latent stages of infection causing apparent brain pathologies in infected rodents and both immuno-compromised and immuno-competent humans. In immuno-competent individuals, behavioural disorders are primarily related to the latent stages of the illness. Behavioural/mental disorders that include schizophrenia, mood disorders, personality changes and cognitive impairments may be related to infection with $T$. gondii. Evidence for a behavioural effect of $T$. gondii comes from observational reports in animal models and controlled behavioural analysis in humans. Indirect clues of infection also come from raised seroprevalence or serotitres of antitoxoplasma antibodies among those with mental disorders. The pathophysiologic mechanism through which $T$. gondii may exert its effect is not clear, but direct impact on the brain and changes in neuroimmunomodulation, neurotransmission and some gene-environment interactions are postulated. Conclusion - There is evidence supporting a potential role of $T$. gondii infection in the onset of some behavioural disorders. Confirmation of such a role would prove a significant breakthrough in the search for the aetiology, treatment and prevention of behavioural disorders, such as schizophrenia. However, the associations remain preliminary.
\end{abstract}

Keywords: toxoplasmosis, Toxoplasma gondii, behavioural disorder, personality changes, schizophrenia, mood disorders, review

Mental and behavioural disorders have major public health impact. They occur commonly, affecting 10-16\% of the population in any given year (Demyttenaere et al. 2004, Wang et al. 2007). Mental and behavioural disorders are also associated with high level of individual distress, disability and premature mortality. The cost in terms of lost productivity and use of health services is substantial and these disorders contribute to $12 \%$ of the global disease burden (World Health Organisation 2001), which is greater than the burden caused by cardiovascular conditions or malignant neoplasms (World Health Organisation 2001, Kohn et al. 2004). The treatment for mental and behavioural disorders is mostly symptomatic because their aetiology is still obscure. The current consensus is behavioural disorders are multifactorial in causation and relate to genetic predisposition and environmental mediation. There is increasing interest in the hypothesis that infectious agents, such as Toxoplasma gondii, herpes simplex virus, cytomegalovirus and influenza virus, may play an aetiological role in some mental and behavioural disorders. The most extensive work has gone into investigating the association between schizophrenia and T. gondii with a special issue dedicated recently to this subject in the Schizophrenia Bulletin (Schizophrenia Bulletin Vol. 33, no. 3, 2007).

Toxoplasma gondii is a neurotropic protozoan parasite that was characterised at the turn of the $20^{\text {th }}$ century and later associated with a series of congenital sensory and neurological conditions (Wolf et al. 1939, Torrey et al. 2007). Toxoplasma gondii infection is widespread and is reported to affect up to a third of the world's population (Montoya and Liesenfeld 2004). Although the definitive hosts are felines such as cats, $T$. gondii affects almost all warm-blooded animals including humans. In humans, infection is mainly acquired by ingestion of contaminated food or water or through eating undercooked or raw meat that contains tissue cysts (Montoya and Liesenfeld 2004). Overt and serious disease from toxoplasmosis occurs in immuno-compromised hosts. In some patients cervical lymphadenopathy and ocular disease may occur, but in most immuno-competent individuals infection is subclinical (Montoya and Liesenfeld 2004). 
Its neurotropic nature and other characteristics make T. gondii an attractive candidate as a potential causative agent for mental and behavioural disorders. Toxoplasma gondii uses a complex mechanism to gain access to the brain. Once access has been gained, it invades various brain cells, including astrocytes and neurons, where it forms cysts (Carruthers and Suzuki 2007). Toxoplasma gondii can then establish a continuous infection within the central nervous system (CNS), manipulate intermediate host behaviour, and can cause neurological and psychiatric symptoms in some infected individuals (Holliman 1997, Webster et al. 2006). Various immunological reactions and reactivation mechanisms operate, which are also linked with the onset of behavioural disorders in humans. Based on a narrative review, this article attempts to provide an overview of the evidence that indicates the potential aetiological importance of $T$. gondii in behavioural disorders and the mechanisms through which any effect of $T$. gondii might be mediated.

\section{Method}

This is a narrative review of studies that reported on the association of $T$. gondii with mental and behavioural disorders or personality changes in humans. Reports on animal models were not systematically reviewed but were referred to when relevant to demonstrating the effect of $T$. gondii on human behaviour. The main data sources for the review were Medline, PsycINFO, PubMed, Google Scholar and Google. References of identified articles were also used. We used the following key terms to identify the studies: toxoplasmosis; Toxoplasma gondii; mood disorder; affective disorder; bipolar disorder; depression; psychotic disorder; schizophrenia; personality disorder; obsessive compulsive disorder; anxiety disorder; phobic disorder; and mental and behavioural disorder. Relevant articles (including reviews, original reports, case reports) from 1950 to the present (Jan 2010) were included. The approach to gathering the literature was systematic. However, as there has been a recent systematic review on the association of schizophrenia with $T$. gondii, it was felt that an attempt to do a systematic review here would only duplicate the work with limited additional benefit. Additionally, reports exploring the aetiological relevance of $T$. gondii more directly and more hypothesis driven explorations do not yield themselves to a meta-analysis although are included in the review. However, a limited data synthesis was made when appropriate. For example, in reviews where total numbers of cases were not computed, this was done. Odds ratios were calculated to show the magnitude of the association in individual case-control studies or for some reviews.

\section{Results}

For the data-based reports, 104 studies were initially identified for inclusion. Of these, 24 studies were of adequate methodological quality for inclusion (Table 1). Three of these studies were reviews (Torrey and Yolken 2003, Torrey et al. 2007, Zhu et al. 2007a); two were case reports (Brynska et al. 2001, Kara and Misra 2004) and most of the remaining studies were original case-control studies focusing on the seroprevalence of $T$. gondii among patients with various psychiatric disorder. In the current review, original studies included in the review by Torrey et al. (2007) were included as part of that review. The focus here was on studies published since the Torrey et al. review (2007). We also included studies dealing with disorders other than schizophrenia. Four unpublished reports and other hypothetical studies exploring the aetiological relevance of $T$. gondii were also included.

The review found that toxoplasmosis was associated with several mental and behavioural disorders as well as personality changes. Most of the identified reports focused on schizophrenia, but other disorders considered included bipolar disorder, depressive disorder and obsessive compulsive disorder. Toxoplasmosis was also linked with subtle behavioural and personality changes and with some risk behaviour, for example, suicidality and involvement in road traffic accidents.

Table 1. Selected studies included in review.

\begin{tabular}{|c|c|c|c|c|}
\hline Author & Year & Design & Sample size & Findings \\
\hline $\begin{array}{l}\text { Tedla et al. } \\
\text { unpublished }\end{array}$ & 2010 & Case-control & $\begin{array}{l}216 \text { schizophrenia } \\
80 \text { controls }\end{array}$ & $\begin{array}{l}\text { Seroprevalence in cases }=97.7 \% \\
\text { OR }(\mathbf{9 5} \% \mathbf{C I})=\mathbf{4 . 7}(\mathbf{9 5} \% \mathbf{C I}=\mathbf{1 . 5}, \mathbf{1 5 . 1})\end{array}$ \\
\hline Xiao and Buka & 2009 & $\begin{array}{l}\text { Case-control } \\
\text { (cases with schizophrenia) }\end{array}$ & $\begin{array}{l}219 \text { cases } \\
618 \text { controls }\end{array}$ & Strain-specific elevated risk for schizophrenia. \\
\hline $\begin{array}{l}\text { Dogruman-Al } \\
\text { et al. }\end{array}$ & 2009 & $\begin{array}{l}\text { Case-control } \\
\text { (cases with schizophrenia) }\end{array}$ & $\begin{array}{l}88 \text { cases } \\
88 \text { controls }\end{array}$ & $\begin{array}{l}\text { Seroprevalence higher in cases }(47.7 \%) \text { than controls }(20.4 \%) \\
* \mathbf{O R} ; \mathbf{9 5 \%} \mathbf{C I}=\mathbf{3 . 5 5} ; \mathbf{1 . 8 2}, \mathbf{6 . 9 1}\end{array}$ \\
\hline Brown et al. & 2009 & $\begin{array}{l}\text { Case-control } \\
\text { (cases with schizophrenia } \\
\text { and schizophrenia spectrum) }\end{array}$ & $\begin{array}{l}\text { Exposed to } T \text {. gondii during } \\
\text { pregnancy (cases) }=26 \\
\text { Unexposed (controls) }=24\end{array}$ & $\begin{array}{l}\text { Executive dysfunction higher among } \\
\text { those with gestational exposure. }\end{array}$ \\
\hline Arling et al. & 2009 & $\begin{array}{l}\text { Case-control } \\
\text { (cases with recurrent } \\
\text { mood disorders with his- } \\
\text { tory of self harm; controls: } \\
\text { with depression but no } \\
\text { self harm or normal) }\end{array}$ & $\begin{array}{l}99 \text { cases } \\
119 \text { controls with } \\
\text { depression } \\
39 \text { healthy controls }\end{array}$ & Higher $T$. gondii IgG antibody titres among cases. \\
\hline Tamer et al. & 2008 & $\begin{array}{l}\text { Case-control } \\
\text { (cases with schizophrenia) }\end{array}$ & $\begin{array}{l}40 \text { cases } \\
37 \text { controls }\end{array}$ & $\begin{array}{l}\text { Seroprevalence: } 40 \% \text { in cases, } 13.5 \% \text { in controls. } \\
\text { *OR; } \mathbf{9 5 \%} \mathbf{C I}=\mathbf{4 . 2 7} ; \mathbf{1 . 3 7} \mathbf{1 3 . 2 8}\end{array}$ \\
\hline
\end{tabular}


Table 1. Continued.

\begin{tabular}{|c|c|c|}
\hline Author & Year & Design \\
\hline Niebuhr et al. & 2008 & Case-control \\
\hline Zhu et al. & $2007 \mathrm{a}$ & $\begin{array}{l}\text { Review of } 15 \text { serologi- } \\
\text { cal surveys in China } \\
\text { (original studies case- } \\
\text { control except two; } \\
\text { cases with psychosis) }\end{array}$ \\
\hline Mortensen et al. & 2007 & $\begin{array}{l}\text { Case-control } \\
\text { (cases: patients with schizo- } \\
\text { phrenia and mood disorders } \\
\text { with maternal exposure } \\
\text { to T. gondii infection) }\end{array}$ \\
\hline
\end{tabular}

Hinze-Selch et al. 2007 Case-control

Cetinkaya et al. 2007 Case-control

Amminger et al. 2007 Cross-sectional

Torrey et al.

2007 Systematic review

Alvarado-

Esquivel et al.

2006 Case-control

Skallová et al. 2005 Case-control
El-Sahn et al. $\quad 2005$ Case-control
Bachmann et al. 2005 Cross-sectional

Brown et al.

2005 Nested case-control (cases with schizophrenia spectrum disorders)

Thomas et al. 2004 Cross-sectional

Leweke et al. 2004 Case-control

Kar and Misra 2004 Case report

Flegr et al. 2003 Cross-sectional

Torrey and Yolken 2003 Systematic review

Brynska et al. 2001 Case report
Sample size

180 cases with

schizophrenia

532 healthy controls

4,269 cases

2,485 controls

186 schizophrenia

258 bipolar disorder and

other mood disorders

31 both diagnosis

$($ Total $=413$ )

2 controls for each case

Cases: schizophrenia $=277$; No difference in seroprevalence.

major depression $=465 \quad$ Serointensity (antibody titre) high in first-episode patients. Controls: 214

100 schizophrenia 50 depression 50 healthy controls

105 individuals considered at "ultra-high risk for psychosis" and aged 14-29

3,873 schizophrenia 7,046 controls

137 from psychiatric inpatient; 180 controls (38 schizophrenia)

290 blood donors ( 85 female); novelty seeking assessed with TCI

75 schizophrenia 85 controls

16 schizophrenia 4 schizoaffective 13 schizophreniform 1 psychosis NOS

63 cases with exposure from pregnancy 123 matched controls 452 farmers (assessed with CIS-R) Toxoplasma IgG in serum and CSF in recent onset schizophrenia

1 with depression

857 military conscripts

4,246 cases with schizophrenia 21,099 controls

2 children with obsessive compulsive disorder
Findings

Antibody level effect on the risk of schizophrenia was raised.

$\mathrm{HR}=1.24 ; 95 \% \mathrm{CI}=1.12,1.37$

Prevalence between $6.37 \%$ and $39.8 \%$. In case-control studies: all significant difference except one study. Overall difference*: $\mathbf{O R}=\mathbf{3 . 4 2} ; \mathbf{9 5} \% \mathrm{CI}=\mathbf{2 . 8 1}, \mathbf{4 . 1 6}$

Patients who later developed schizophrenia had increased risk of having IgG antibodies. Specifically, significant elevation in those in the upper quartile levels: $\mathbf{O R}=\mathbf{1 . 7 9 ;} \mathbf{9 5 \%} \mathbf{C I}=\mathbf{1 . 0 1}, \mathbf{3 . 1 5}$

Serointensity (antibody titre) high in first-episode patients.
Significant difference also among patients older than 45 and higher titres.

For schizophrenia, correlation between titre and leukocyte count as well as CRP values.

Use of antipsychotics with putative immunomodulatory effect was significantly correlated with lower antibody titre.

Seroprevalence: in schizophrenia $=66 \%$; in depression $=24 \%$; in controls $=22 \%$. Difference significant only for schizophrenia. *Schizophrenia versus depression:

OR; $95 \%$ CI $=6.15 ; 2.85,13.27$

Schizophrenia versus healthy controls:

OR; $95 \% \mathrm{CI}=\mathbf{6 . 8 8} ; \mathbf{3 . 1 3}, \mathbf{1 5 . 1 2}$

vere positive psychotic symptoms

23 studies included. Prevalence of toxoplasmosis was elevated: the combined odds ratio $95 \%$ CI $2.73(2.10,3.60)$

Prevalence among inpatients $=18.2 \%$;

in controls $=8.9 \%$; in schizophrenia $=26.3 \%$.

*Difference against controls significant; difference for any psychiatric disorder - OR; 95\% CI=2.29; 1.17, 4.48; for schizophrenia - OR; $95 \% \mathrm{CI}=1.51,8.88$

Toxoplasmosis associated with reduced novelty seeking.

Seroprevalence: in schizophrenia $=80 \%$; in controls $=52.9 \%$. *Significant difference $=\mathbf{O R}$; 95\% CI $=3.56 ; 1.75,7.22$

PANSS total score on admission related to levels in IgG antibody.

Elevated maternal Toxoplasma IgG antibody titres $\mathrm{OR}=\mathbf{2 . 6 1} ; \mathbf{9 5 \%} \mathrm{CI}=\mathbf{1 . 0 0}, \mathbf{6 . 8 2}$

Seroprevalence $=45 \%$.

Infection not significantly associated with psychiatric morbidity.

Untreated individuals had significantly raised levels of serum and CSF IgG levels compared to controls; individuals on treatment had lower levels; antibody levels in treated individuals did not differ significantly from controls.

Poor initial response to antidepressants; response to antidepressant improved only with adequate treatment of the toxoplasmosis.

Novelty seeking measured with TCI was lower in those positive for toxoplasmosis. Also lower was score for 3 of the subscores (impulsiveness, extravagance and disorderliness).

Seroprevalence: in cases $=48.1 \%$; in controls $=26.0 \%$.

Significant association with schizophrenia.

OR; 95\% CI $=2.63 ; 2.46,2.81$

Pharmacological therapy of toxoplasmosis without additional psychopharmacological intervention lead to remission or marked improvement in both cases.
Higher IgG antibodies associated with more se-

*Calculated by the present authors. Calculation excludes the two studies where there were no controls. Abbreviations: CI - confidence interval; CIS-R - Clinical Interview Schedule-Revised; CSF - cerebrospinal fluid; HR - hazard ratio; NOS - not otherwise specified; OR - odds ratio; PANSS - Positive and Negative Syndrome Scale; TCI - Temperament and Character Inventory. 


\section{Schizophrenia}

Schizophrenia is one of the most severe psychiatric syndromes. It has a pervasive impact on the affected person's day-to-day life and functioning. With onset often in young adulthood, schizophrenia is characterised by impairment in multiple domains of mental functioning, specifically thought processing, perception, cognition, mood and psychomotor behaviour. These multiple impairments mean that it is also more difficult to model the disorder in animals. Its aetiology, as in most psychiatric disorders, is undetermined but is believed to be a result of the interaction of developmentally predisposing factors (both genetic, neurodevelopmental and environmental) with mediating environmental factors. Such mediating environmental factors are postulated to include infectious agents such as T. gondii. Epidemiological association studies and limited clinical data support the involvement of $T$. gondii.

Epidemiological data relates to the finding of a high seroprevalence of $T$. gondii in patients with schizophrenia compared with healthy controls. The main findings are summarised in Table 1. The seroprevalence for immunoglobulin $\mathrm{G}(\mathrm{IgG})$ antibodies of $T$. gondii were as high as $97 \%$ in some settings. With few exceptions, the seroprevalence was significantly higher among cases with schizophrenia compared to controls. A recent metaanalytic review confirmed this association (Torrey et al. 2007). This report was based on 23 studies that included defined control groups. The studies were identified through a broad search of literature written in English and other languages. A total of 3,873 patients and 7,046 controls were involved. The report confirmed the increased prevalence of $T$. gondii antibodies among patients with schizophrenia compared to healthy controls. The association summarised in terms of an odds ratio (OR) (2.73) was modest but clearly higher than that found for genetic or other environmental risk factors (Torrey et al. 2007), and even cannabis (Zamit et al. 2002). The elevated risk was identified in both first episode patients (OR 2.54) and patients with a variable duration of follow-up $(\mathrm{OR}=2.79)$. The main limitation of the study was that most of the studies involved more severely ill patients recruited through hospital-based samples. Community-based and treatment-naïve patients were not included. The actual implication of omission of the milder forms of the disorder is unclear, although among community-based participants from Ethiopia the seroprevalence for $T$. gondii was even higher ( $\mathrm{n}=80 / 91)$ (Shibre et al. 2009). A recent study in this community setting based on sera from 216 individuals with schizophrenia and 80 unaffected controls reported a $97.7 \%$ seroprevalence among patients with schizophrenia (Tedla et al. 2010, unpublished). The adjusted odds of having positive IgG was 4.7 ( $95 \% \mathrm{CI}=1.5,15.1)$. Although this is a high rate of seroprevalence, the rate was also high among the unaffected individuals (87.3\%). However, it is not clear if these controls had other psychiatric conditions other than schizophrenia.
Numerous studies have been conducted in various regions of China in patients with psychosis. A study comparing patients with first episode schizophrenia $(n=600)$, affective disorders $(n=600)$ and cases with physical ailment and healthy controls $(n=400)$ reported significantly elevated seroprevalence among patients with schizophrenia (Wang et al. 2006). The study found higher seroprevalence among first episode patients against all three comparison groups. A review of studies conducted in various regions of China (Zhu et al. 2007a, b, Zhu 2009) to determine the association of psychosis with toxoplasmosis reported a 3-5 times increase in the prevalence of $T$. gondii infection among psychiatric inpatients compared with healthy controls. In the latest report (Zhu 2009) nine studies were reviewed, and in the first report (Zhu et al. 2007a) 15 studies were reviewed.

Development of schizophrenia was also demonstrated among those with early-life infection with $T$. gondii. In this regard both in utero exposure and early postnatal exposure were found to be associated with an increased risk of schizophrenia (Brown et al. 2005, Mortensen et al. 2007a, b). In another study, serological evidence of T. gondii was also associated with increased risk of dying of natural causes during a five year follow-up (Dickerson et al. 2007). There may be other potential explanations for the increased mortality; however, no socio-demographic or clinical features predicted seropositivity.

Some case reports have indicated that $T$. gondii encephalitis may manifest with symptoms akin to those of schizophrenia or other psychotic disorders (Torrey and Yolken 2003). Studies in patients with AIDS and toxoplasmic encephalitis have reported a high proportion of cases with behavioural manifestations that included delusions, thought disorder and auditory hallucinations (Israelski and Remington 1988, Torrey and Yolken 2003).

Because most studies report on seroprevalence of toxoplasmosis among patients who have already developed schizophrenia, the direction of causality remains one of the main areas of uncertainty. A recent study tried to provide some answer to this question by measuring sera of individuals collected before a diagnosis of schizophrenia was made (Niebuhr et al. 2008). In this case-control study using 180 patients and controls (3:1 ratio), the authors reported a significantly elevated risk of $\mathrm{IgG}$ seroprevalence to $T$. gondii among individuals diagnosed with schizophrenia (hazard ratio of 1.24). As schizophrenia is an insidious illness, the question of whether the blood samples were collected before the onset of the illness is difficult to answer, but this is an interesting exploration that should be followed by other larger-scale studies.

The conclusion that is to be drawn from all the studies described so far is that some cases of schizophrenia may be caused by infection with $T$. gondii and that such infection may also have an impact on the longer-term outcome of the disorder. Further implications of these findings are detailed in the discussion section. 


\section{Affective disorders}

Evidence for a role of $T$. gondii infection in the causation of mood disorders is less extensive but indicative. Indirect evidence comes from intervention studies. Haloperidol, which is an effective medication during the acute phase of a manic illness, has been found to be a potent inhibitor of the growth of $T$. gondii in cell culture (Jones-Brando et al. 2003). Valproic acid, an anticonvulsant drug used as one of the first-line mood stabiliser drugs, is also found to be an effective inhibitor of $T$. gondii growth at relatively low concentrations (Jones-Brando et al. 2003). In a rodent model, antipsychotic drugs have also been proven to be effective in preventing the behavioural alterations, specifically the loss of the innate aversion to cats (Webster et al. 2006). Observations in some animals (pigs, meerkats and the red-necked wallaby) with "acute and peracute" toxoplasmosis have demonstrated depressive and neurological symptoms (Adkesson et al. 2007, Basso et al. 2009, Kim et al. 2009). The incidence of T. gondii infection based on serological evidence has also been reported to be higher in patients with "manic depressive illness"; a collective name for both depressive and bipolar disorder (Delgado and Rodriguez 1980). In a community-based study from Ethiopia involving 199 patients with bipolar disorder and 80 controls (Tedla et al. 2010, unpublished), patients with bipolar disorder were found to have increased seroprevalence (adjusted OR; 95\% CI=3.0; 1.1, 8.6).

Other case reports are also consistent with the above observations. In a series of case reports of patients presenting with acute toxoplasmic encephalitis, one patient was reported to have nihilistic delusions consistent with psychotic depression (Torrey and Yolken 2003). A case presenting with symptoms consistent with depression who failed to respond to antidepressants did so after adequate treatment for the $T$. gondii infection (Kar and Misra 2004). The tendency of mice to behave in a potentially self-destructive way following $T$. gondii infection may also be construed as depressive. A recent study conducted with this assumption in mind compared T. gondii antibody titres of those with recurrent mood disorder and suicide attempt against those with mood disorder but without history of suicide attempt. The study reported significantly higher antibody titres among those with a history of suicide attempt (Arling et al. 2009). However, seroprevalence was not different between the groups. In this particular study, there was no difference in seroprevalence between those with depressive disorder and bipolar disorder or between those with psychiatric diagnosis and those with no such diagnosis. The latter finding may have occurred due to the low power of the study given the very small number of participants without mood disorder. A study that compared a relatively large number of patients with affective disorder $(n=600)$ in China against patients with physical disease and healthy controls $(n=400)$ did not find significantly elevated seroprevalence among patients with affective disorder. However, a larger proportion of patients with affective disorder (6.7\%) manifested $\mathrm{IgG}$ reactivity compared to healthy controls $(3.0 \%)$ and those with physical disease (4.5\%) (Wang et al. 2006).

\section{Other behavioural changes and disorders}

Toxoplasma gondii infection has also been associated with obsessive-compulsive disorder. A case report presented a report of children manifesting obsessivecompulsive symptoms who were later diagnosed with toxoplasmosis (Brynska 2001). Treatment of the infection improved the obsessive-compulsive symptoms. More extensive work has also gone into the determination of the impact of $T$. gondii infection on the behaviour of apparently healthy participants. Thus the impact of toxoplasmosis on personality profiles was reviewed recently (Flegr 2007). Individuals with latent infections showed different personality profiles to those without infection, which also showed variation between men and women. In a series of comparative studies from the Czech Republic using Cattell's 16 Personality Factor (16 PF) questionnaire and the Cloninger's Temperament and Character Inventory (TCI) personality tests, subtle personality changes were demonstrated. These relatively well-powered studies identified consistent differences between those infected with $T$. gon$d i i$ and uninfected individuals. Men had "lower superego strengths (rule consciousness) and higher vigilance"; as well as being "more expedient, suspicious and jealous". These are clearly factors that have the potential to predispose to substance abuse, anxiety, personality disorders and even psychosis. Women showed almost the opposite behaviour: with higher superego strength and factors that suggested warmth, conscientiousness and moral adherence. But both men and women were found to have more apprehension compared with uninfected controls. Both men and women also showed a decrease in the noveltyseeking factor measured using the TCI in three of the five studies. Among both men (Flegr et al. 1996) and women (Flegr et al. 2000), these differences in personality profile between Toxoplasma-infected and Toxoplasma-free subjects increased with the length of $T$. gondii infection, after the age of subjects was controlled. This might to some extent be relevant to the age of onset of schizophrenia (Carruthers and Suzuki 2007) and the predisposing role of personality to acquiring infection.

Changes in psychomotor behaviour have been demonstrated in animal models as well as in humans. In animal models, change in the risk perception and novelty seeking behaviour of mice has been shown in those infected with T. gondii (Webster et al. 2006, Vyas et al. 2007, Webster 2007). Mice with infection become less risk averse and were attracted to the portion of the apparatus that was cat-treated, which they ordinarily avoid. Such behaviour improved when mice were treated with haloperidol (Webster et al. 2006). Novelty seeking, another characteristic 
behaviour of mice was also reduced when they were infected with $T$. gondii. In humans, cognitive testing demonstrated reduced concentration and prolongation of reaction time. Traffic accidents were also reported to be higher among those with latent $T$. gondii infection (Flegr et al. 2002, Yereli et al. 2006).

\section{Discussion}

Based on existing evidence, the studies summarised so far indicate that $T$. gondii infection may be on the causation pathway of various mental and behavioural disorders. The suggested involvement of $T$. gondii extends from subtle neurobehavioural impairments to severe manifestations of schizophrenia requiring inpatient care. The risk of schizophrenia is elevated among those with in utero exposure and those with exposure to infection during the postnatal period. The likelihood of involvement of $T$. gondii is also demonstrated by the higher seroprevalence of $T$. gondii antibodies among those with schizophrenia compared to controls. This is supported by a recent metaanalytic report (Torrey et al. 2007), the gold standard in the hierarchy of evidence-based clinical practice.

The more subtle behavioural changes are demonstrated in both animal experiments and human observations. These observations are important in so far as they point to the potential role of infectious agents in the causation of mental disorders, which are by and large of undetermined aetiology. But, it is also important to elucidate how such an infection could be on the aetiological pathway of complex disorders, such as schizophrenia. Studies with increasing sophistication have indicated the potential areas for such link.

\section{Mechanism}

Direct effect: Toxoplasma gondii is a neurotropic organism with a complex mechanism of access to the brain where it encysts in neurons, glial cells and astrocytes (Halonen et al. 1996, Carruthers and Suzuki 2007). In chronically infected mice, the cysts were found in multiple areas of the neurons (axons, dendrites and cell body) and were also demonstrated in congenital toxoplasmosis (Ferguson and Hutchison 1987, Sims et al. 1989). Damage is characterised by many foci of enlarging necrosis and microglia nodules. Periaqueductal and periventricular vasculitis, hydrocephalus resulting from obstruction of the aqueduct of Sylvius or foramen of Monro have been demonstrated in infants (Montoya and Liesenfeld 2004). In congenital toxoplasmosis, more intense necrosis occurs in the cortex and basal ganglia, and sometimes in the periventricular areas (Montoya and Liesenfeld 2004). Multiple brain abscesses may occur in severely immunocompromised individuals as a characteristic feature of toxoplasmic encephalitis (Montoya and Liesenfeld 2004). Therefore, $T$. gondii has the potential to effect some im- portant changes in human behaviour through its direct impact on brain tissue. These brain tissue changes may be a result of the direct action of the $T$. gondii or come about as a result of inflammatory responses. Toxoplasma gondii might also incorporate its genome into the cellular deoxyribonucleic acid with the potential of altering brain function or modifying the growing neuronal cell in utero (Fallon 2009). Alternatively T. gondii may effect brain changes by inducing host-determined humoral and cytokine immune responses (Fallon 2009, Henriquez et al. 2009). Activation of pro-inflammatory cytokines or the induction of nitric oxide in the adult brain may lead to neuronal and behavioural dysfunction, or in the developing embryo can lead to inhibited dendritic development in cortical neurons. The immune response may therefore induce a neuropsychiatric condition (Fallon 2009). The cytokines may also influence mood and behaviour by modulating neurotransmission such as the monoamine- and glutamate-dependent systems in the brain (Dunn 2006, Muller and Schwartz 2007a, b). Of additional interest in this regard is the recent discovery of two genes encoding for tyrosine hydroxylase that produces L-DOPA in T. gondii (Gaskell et al. 2007). This enzyme metabolised both phenylalanine and tyrosine with a substrate preference for tyrosine. This finding, in combination with the ability of T. gondii to induce a cytokine response with the potential to modulate neurotransmission systems known to be important in psychotic and mood disorders, makes $T$. gondii a reasonable candidate for causing these disorders.

An intriguing recent report was the association of Rhesus $(\mathrm{RH})$ status with $T$. gondii infection and reaction time (Novotná et al. 2008). Based on a series of studies involving 553 participants $(68.5 \%$ men) and relatively complex analytic procedures, the study reported a significantly prolonged reaction time among Toxoplasma-infected men. Among RH positive men, no such prolongation was demonstrated. However, it was only the heterozygous (Dd genotype) individuals who were protected from Toxoplas$m a$-induced impairment of reaction time. As an important extension of this was the finding of a protective association of $\mathrm{RhD}$ positive status from the increased incidence of traffic accidents even among Toxoplasma-infected military drivers (Flegr et al. 2009). In a prospective study of 3,890 military drivers, a significant interaction of $\mathrm{RhD}$ status and traffic accidents was reported. In a separate analysis of $\mathrm{RhD}$ negative participants, the risk of traffic accident was 2.53 times higher in Toxoplasma-infected compared with non-infected participants. The probability of accidents was increased with the titre of anti-Toxoplas$m a$ antibodies suggesting decrease in risk with duration of Toxoplasma infection. The authors explain the association with RH status in evolutionary terms (Novotná et al. 2008) as well as in terms of the potential involvement of the RH proteins in "the regulation of ion balance in some critical compartment of nerve or muscle tissue" (Flegr et 
al. 2009). This may also have some link with modulation of the inflammatory process.

\section{Hypothesis limitations}

Although the link between some psychiatric disorders and $T$. gondii infection appears plausible, there are numerous difficulties with this proposition. The most obvious problem is the apparent epidemiological inconsistency. Toxoplasma gondii is a ubiquitous parasite and affects nearly a third of the world's population, with a marked (over 8-fold) geographic variation. However, psychiatric disorders so far attributable or associated with $T$. gondii are not so prevalent in high seroprevalence areas and do not exhibit such marked geographic variation in prevalence. Therefore, if $T$. gondii causes psychiatric disorders as proposed, it can only cause such disorders in a fraction of individuals it infects. Moreover, psychiatric disorders are complex conditions that result from multiple interacting factors, one of which may be an infection with $T$. gondii. It has also not been possible to demonstrate a link between infection with $T$. gondii and the timing of onset of these psychiatric conditions. This has only been demonstrated in severely immunodeficient individuals where reactivation of infection resulted in toxoplasmic encephalitis that can be identified in the brain. On the other hand T. gondii is known to induce similar changes in the brain, particularly in congenital toxoplasmosis, to those found in some mental disorders, for example schizophrenia. It is also suggested that early infection can cause lasting changes in neurodevelopment consistent with the neurodevelopmental theory of some disorders such as schizophrenia.

It has also been difficult to demonstrate $T$. gondii in the brain of patients with mental disorders, particularly those investigated in relation to schizophrenia. But this demonstration is not always necessary. Infectious agents may have provided "an aberrant influence at a critical developmental period that exerts long-lasting effects" (Fallon 2009). Causation of psychiatric disorders is determined by a complex interaction between host response genes, T. gondii and the timing in neurodevelopment (Fallon 2009).

Finally, supportive evidence from treatment trials using known medications against toxoplasmosis has not been forthcoming. A six-month double blind randomisedcontrolled trial in Ethiopia using trimethoprim $200 \mathrm{mg} / \mathrm{d}$ added onto standard antipsychotic medication regimes and administered to 91 community patients with chronic schizophrenia did not find any benefit from the add-on trimethoprim (Shibre et al. 2009). A second trial was also conducted in Ethiopia. In this open study, inpatients with schizophrenia were given add-on trimethoprim-sulfamethoxazole $(160 \mathrm{mg} / 800 \mathrm{mg})$. Although patients in the intervention arm showed some improvement at week 8 , there was no difference at week 16 (Desta et al. unpublished). A study from the USA used adjunctive azithromycin (Dickerson 2009). In this study, azithromycin was administered to 28 adults with chronic schizophrenia (mean duration of illness of 22.3 years) and a significant level of symptomatology having a total score of $71.6( \pm 11.4)$ according to the Positive and Negative Syndrome Scale (PANSS) (Kay et al. 1987). Patients receiving the trial medication did not show any differential benefit. However, the interpretation of these several negative findings is not easy. First, all of the studies were underpowered to detect any meaningful effect of intervention. Second, participants in all the studies included were chronically and severely ill and so any additional modest effect is much less likely to have been detected. Third, as discussed above, the effect of $T$. gondii is likely to be early in life at the neurodevelopmental stage rather than at the age of actual illness onset. These early changes are likely to be irreversible. Therefore, given the likely timing of exposure and the complex interaction of $T$. gondii infection with the human host, intervention studies may not be the ideal method to prove the link between behavioural disorders and $T$. gondii infection. On the other hand, although with ethical and practical challenges, prophylactic intervention early in childhood among a high risk subgroup may be considered. Such high risk groups may be those with a family history of a severe mental disorder such as schizophrenia or birth trauma. Intervention nearer the time of onset of illness or during prodrome may also be feasible but additional markers may be required.

\section{Conclusions}

The proposition that in some cases $T$. gondii infection can cause mental and behavioural disorders seems plausible. This is supported by evidence from animal models that show association between infection with $T$. gondii and distinct behavioural changes that improve with medications used to treat some mental disorders. In humans, infection with $T$. gondii is associated with various mental and behavioural disorders ranging from subtle personality changes to severe disorders like schizophrenia. These findings are also supported by plausible propositions of mechanisms that may lead to these mental and behavioural changes. But further confirmation of these findings is required. For example, the clinical relevance of the discovery of genes encoding for tyrosine hydroxylase in $T$. gondii has to be confirmed. If T. gondii is shown to be a cause of mental disorders even in some cases, it will constitute a major breakthrough in understanding the aetiology and pathophysiological mechanisms of psychiatric disorders, as well as their treatment and prevention.

Acknowledgement. We are grateful to Dr. Charlotte Hanlon for commenting on manuscript. 
Adkesson M.J., Gorman M.E., Hsiao V., Whittington J.K., LANGAN J.N. 2007: Toxoplasma gondii inclusions in peripheral blood leukocytes of red-necked wallaby (Macropus rufogriseus). Vet. Clin. Pathol. 36: 97-100.

Alvarado-Esquivel C., Alanis-Quinones O.P., ArreolaValenzuela M.A., Rodriguez-Briones A., Piedra-Nevarez L.J., Duran-Morales E., Estrada-Martinez S., MartinezGarcia S.A., Liesenfeld O. 2006: Seroepidemiology of Toxoplasma gondii infection in psychiatric inpatients in a northern Mexican city. BMC Infect. Dis. 6: e178.

Amminger G.P., McGorry P.D., Berger G.E., Wade D., Yung A.R., Phillips L.J., Harrigan S.M., Francey S.M., Yolken R.H. 2007: Antibodies to infectious agents in individuals at ultra-high risk for psychosis. Biol. Psychiat. 61: 1215-1217.

Arling T., Yolken R.H., Lapidus M., Langenberg P., Dickerson F.B., Zimmerman S.A., Balis T., Cabassa J.A., ScranDis D.A., Tonelli L.H., Postolache T.T. 2009: Toxoplasma gondii antibody titers and history of suicide attempts in patients with recurrent mood disorders. J. Nerv. Ment. Dis. 197: 905-908.

Bachmann S., Schroder J., Bottmer C., Torrey E.F., Yolken R.H. 2005: Psychopathology in first-episode schizophrenia and antibodies to Toxoplasma gondii. Psychopathol. 38: 87-90.

Basso W., Moré G., Quiroga M.A., Pardini L., Bacigalupe D., Venturini L., Valenzuela M.C., Balducchi D., Maksimov P., Schares G., Venturini M.C. 2009: Isolation and molecular characterization of Toxoplasma gondii from captive slendertailed meerkats (Suricata suricatta) with fatal toxoplasmosis in Argentina. Vet. Parasitol. 161: 201-206.

Brown A.S., Schaefer C.A., Quesenberry C.P., Jr., Liu L., Babulas V.P., Susser E.S. 2005: Maternal exposure to toxoplasmosis and risk of schizophrenia in adult offspring. Am. J. Psychiat. 162: 767-773.

Brown A.S., Vinogradov S., Kremen W.S., Poole J.H., Deicken R.F., Penner J.D., McKeague I.W., Kochetkova A., Kern D., Schaefer C.A. 2009: Prenatal exposure to maternal infection and executive dysfunction in adult schizophrenia. Am. J. Psychiat. 166: 683-690.

BRYNSKA A. 2001: Obsessive-compulsive disorder and acquired toxoplasmosis in two children. Eur. Child Adolesc. Psychiat. 10: 200-204.

Carruthers V.B., Suzuki Y. 2007: Effects of Toxoplasma gondii infection on the brain. Schizophr. Bull. 33: 745-751.

Cetinkaya Z., Yazar S., Gecici O., Namli M.N. 2007: AntiToxoplasma gondii antibodies in patients with schizophrenia - preliminary findings in a Turkish sample. Schizophr. Bull. 33: 789-791

Delgado G.G., Rodriguez P.E. 1980: Reactivity of toxoplasmin intradermal test in neurotic and manic-depressive patients. Rev. Cuba. Med. Trop. 32: 35-39.

Demyttenaere K., Bruffaerts R., Posada-Villa J., Gasquet I., Kovess V., Lepine J.P., Angermeyer M.C., Bernert S., de Girolamo G., Morosini P., Polidori G., Kikkawa T., Kawakami N., Ono Y., Takeshima T., Uda H., Karam E.G., Fayyad J.A., Karam A.N., Mneimneh Z.N., Medina-Mora M.E., Borges G., Lara C., de Graaf R., Ormel J., Gureje O., Shen Y., Huang Y., Zhang M., Alonso J., Haro J.M., VIlagut G., Bromet E.J., Gluzman S., Webb C., Kessler R.C., Merikangas K.R., Anthony J.C., Von Korff M.R., Wang P.S., Brugha T.S., Aguilar-Gaxiola S., Lee S., Heeringa S., Pennell B.E., Zaslavsky A.M., Ustun T.B., Chatterji S.; Who World Mental Health Survey Consortium 2004:
Prevalence, severity, and unmet need for treatment of mental disorders in the World Health Organization World Mental Health Surveys. JAMA (J. Am. Med. Assoc.) 291: 2581-2590.

Dickerson F., Boronow J., Stallings C., Origoni A., Yolken R. 2007: Toxoplasma gondii in individuals with schizophrenia: association with clinical and demographic factors and with mortality. Schizophr. Bull. 33: 737-740.

Dickerson F.B., Stallings C.R., Boronow J.J., Origoni A.E., Yolken R.H. 2009: A double-blind trial of adjunctive azithromycin in individuals with schizophrenia who are seropositive for Toxoplasma gondii. Schizophr. Res. 112: 198-199.

Dogruman-Al F., Asian S., Yalcin S., Kustimur S., Turk S. 2009: A possible relationship between Toxoplasma gondii and schizophrenia: a seroprevalence study. Int. J. Psychiat. Clin. Pract. 13: 82-87.

DunN A.J. 2006: Effects of cytokines and infections on brain neurochemistry. Clin. Neurosci. Res. 6: 52-68.

El-Sahn A.A., Shatat H.Z., Ghitany E.M. 2005: Seropositivity of toxoplasmosis in patients with schizophrenia. J. Egypt. Public Health Assoc. 80: 509-524.

FALLON B.A. 2009: Neuropsychiatric aspects of other infectious diseases (Non-HIV). In: B.J. Sadock, V.A. Sadock and P. Ruiz (Eds.), Kaplan \& Sadock's Comprehensive Textbook of Psychiatry. 9th Ed., Vol 1. Philadelphia, Lippincott Williams \& Wilkins, $532 \mathrm{pp}$.

Ferguson D.J., Hutchison W.M. 1987: The host-parasite relationship of Toxoplasma gondii in the brains of chronically infected mice. Virchows Arch. A. Pathol. Anat. Histopathol. 411: 39-43.

FLEGR J. 2007: Effects of Toxoplasma on human behavior. Schizophr. Bull. 33: 757-760.

Flegr J., Havlíček J., Kodym P., Malý M., Šmahel Z. 2002: Increased risk of traffic accidents in subjects with latent toxoplasmosis: a retrospective case-control study. BMC Infect. Dis. 2: e11.

Flegr J., Klose J., Novotná M., Berenreitterová M., Havlíček J. 2009: Increased incidence of traffic accidents in Toxoplasmainfected military drivers and protective effect $\mathrm{RhD}$ molecule revealed by a large-scale prospective cohort study. BMC Infect. Dis. 9: e72.

Flegr J., Kodym P., Tolarová V. 2000: Correlation of duration of latent Toxoplasma gondii infection with personality changes in women. Biol. Psychol. 53: 57-68.

Flegr J., Preiss M., Klose J., Havlíček J., Vitáková M., Kodym P. 2003: Decreased level of psychobiological factor novelty seeking and lower intelligence in men latently infected with the protozoan parasite Toxoplasma gondii. Dopamine, a missing link between schizophrenia and toxoplasmosis? Biol. Psychol. 63: 253-268.

Flegr J., Zitková S., Kodym P., Frynta D. 1996: Induction of changes in human behaviour by the parasitic protozoan Toxoplasma gondii. Parasitology 113: 49-54.

Gaskell E.A., Smith J.E., Pinney J.W., Westhead D.R., McCoNKEY G.A. 2009: A unique dual activity amino acid hydroxylase in Toxoplasma gondii. PLoS ONE 4: e4801.

Halonen S.K., Lyman W.D., Chiu F.C. 1996: Growth and development of Toxoplasma gondii in human neurons and astrocytes. J. Neuropathol. Exp. Neurol. 55: 1150-1156.

Henriquez S.A., Brett R., Alexander J., Pratt J., Roberts C.W. 2009: Neuropsychiatric disease and Toxoplasma gondii infection. Neuroimmunomodulation 16: 122-133.

Hinze-Selch D., Däubener W., Eggert L., Erdag S., StoltenBERG R., WILMS S. 2007: A controlled prospective study of 
Toxoplasma gondii infection in individuals with schizophrenia: beyond seroprevalence. Schizophr. Bull. 33: 782-788.

Holliman R.E. 1997: Toxoplasmosis, behavior and personality. J. Infect. 35: 105-110.

Israelski D.M., Remington J.S. 1988: Toxoplasmic encephalitis in patients with AIDS. Infect. Dis. Clin. North Am. 2: 429-445.

Jones-Brando L., Torrey E.F., Yolken R. 2003: Drugs used in the treatment of schizophrenia and bipolar disorder inhibit the replication of Toxoplasma gondii. Schizophr. Res. 62: 237-244.

Kar N., Misra B. 2004: Toxoplasma seropositivity and depression: a case report. BMC Psychiat. 4: el.

Kay S.R., Fiszbein A., Opler L.A. 1987: The positive and negative syndrome scale (PANSS) for schizophrenia. Schizophr. Bull. 13: 261-276.

Kim J.H., Kang K.I., Kang W.C., Sohn H.J., Jean Y.H., Park B.K., Kıм Y., Kıм D.Y. 2009: Porcine abortion outbreak associated with Toxoplasma gondii in Jeju Island, Korea. J. Vet. Sci. 10: 147-151.

Kohn R., Saxena S., Levav I., Saraceno B. 2004: Treatment gap in mental health care. Bull. WHO 82: 858-866.

Leweke F.M., Gerth C.W., Koethe D., Klosterkotter J., Ruslanova I., Krivogorsky B., Torrey E.F., Yolken R.H. 2004: Antibodies to infectious agents in individuals with recent onset schizophrenia. Eur. Arch. Psychiat. Clin. Neurosci. 254: 4-8.

Montoya J.G., Liesenfeld O. 2004: Toxoplasmosis. Lancet 363: 1965-1976.

Mortensen P.B., Norgaard-Pedersen B., Waltoft B.L., SoRensen T.L., Hougaard D., Yolken R.H. 2007a: Early infection of Toxoplasma gondii and the later development of schizophrenia. Schizophr. Bull. 33: 741-744.

Mortensen P.B., Norgaard-Pedersen B., Waltoft B.L., SoRensen T.L., Hougaard D., Torrey E.F., Yolken R.H. 2007b: Toxoplasma gondii as a risk factor for early-onset schizophrenia: analysis of filter paper blood samples obtained at birth. Biol. Psychiat. 61: 688-693.

Muller N., Schwartz M.J. 2007a: The immune-mediated alteration of serotonin and glutamate towards an integrated view of depression. Mol. Psychiat. 12: 988-1000.

Muller N., Schwartz M.J. 2007b: The immunological basis of glutamatergic disturbance in schizophrenia: towards an integrated view. J. Neural Transm. 72 (Suppl.): 269-280.

Niebuhr D.W., Millikan A.M., Cowan C.N., Yolken R., Li Y., Weber N.S. 2008: Selected infectious agents and risk of schizophrenia among U.S. military personnel. Am. J. Psychiat. 165: 99-106.

Novotná M., Havlíček J., Smith A.P., Kolbeková P., Skallová A., Klose J., Gašová Z., Písačka M., Sechovská M., Flegr J. 2008: Toxoplasma and reaction time: role of toxoplasmosis in the origin, preservation and geographical distribution of Rh blood group polymorphism. Parasitology 135: 1253-1261.

Shibre T., Alem A., Abdulahi A., Araya M., Beyero T., Medhin G., Deyassa N., Negash N., Nigatu A., Kebede D., FeKADU A. 2009: Trimethoprim as adjuvant treatment in schizophrenia: A double-blind, randomized, placebo-controlled clinical trial. Schizophr. Bull. doi: 10.1093/schbul/sbn191.

Sims T.A., Hay J., Talbot I.C. 1989: An electron microscope and immunohistochemical study of the intracellular Toxoplasma tissue cysts within the brain of mice with congenital toxoplasmosis. Br. J. Exp. Pathol. 70: 317-325.

Skallová A., Novotná M., Kolbeková P., Gašová Z., Veselý V., Sechovská M., Flegr J. 2005: Decreased level of novelty seeking in blood donors infected with Toxoplasma. Neuroendocrinol. Lett. 26: 480-486.

Tamer G.S., Dundar D., Yalug I., Caliskan S., Yazar S., AKer A. 2008: The schizophrenia and Toxoplasma gondii connection: infectious, immune or both? Adv. Ther. 25: 703-709.

Thomas H.V., Thomas D.R., Salmon R.L., Lewis G., Smith A.P. 2004: Toxoplasma and Coxiella infection and psychiatric morbidity: a retrospective cohort analysis. BMC Psychiat. 4: e32.

Torrey E.F., Bartko J.J., Lun Z.-R., Yolken R.H. 2007: Antibodies to Toxoplasma gondii in patients with schizophrenia: a meta-analysis. Schizophr. Bull. 33: 729-736.

Torrey E.F., Yolken R.H. 2003: Toxoplasma gondii and schizophrenia. Emerg. Infect. Dis. 9: 1375-1380.

Vyas A., Kim S.K., Giacomini N., Boothroyd J.C., Sapolsky R.M. 2007: Behavioral changes induced by T. gondii infection of rodents are highly specific to aversion of cat odors. Proc. Natl. Acad. Sci. U. S. A. 104: 6442-6447.

Wang H.-L., Wang G.-H., Li Q.-Y., Shu C., Jiang M.S., Guo Y. 2006: Prevalence of Toxoplasma infection in first-episode schizophrenia and comparison between Toxoplasma-seropositive and Toxoplasma-seronegative schizophrenia. Acta Psychiatr. Scand. 114: 40-48.

Wang P.S., Aguilar-Gaxiola S., Alonso J., Angermeyer M.C., Borges G., Bromet E.J., Bruffaerts R., de Girolamo G., de Graaf R., Gureje O., Haro J.M., Karam E.G., Kessler R.C., Kovess V., Lane M.C., Lee S., Levinson D., Ono Y., Petukhova M., Posada-Villa J., Seedat S., Wells J.E. 2007: Use of mental health services for anxiety, mood, and substance disorders in 17 countries in the WHO world mental health surveys. Lancet 370: 841-850.

WeBSTER J.P. 2007: The effect of Toxoplasma gondii on animal behavior: playing cat and mouse. Schizophr. Bull. 33: 752-756.

Webster J.P., Lamberton P.H., Donnelly C.A., Torrey E.F. 2006: Parasites as causative agents of human affective disorders? The impact of anti-psychotic, mood-stabilizer and antiparasite medication on Toxoplasma gondii's ability to alter host behaviour. Proc. R. Soc. Lond., Ser. B, 273: 1023-1030.

Wolf A., Cowen D., Paige B.H. 1939: Human toxoplasmosis. Science 89: 226-227.

World Health Organisation 2001: World Health Report 2001. Mental Health: New Understanding, New Hope. World Health Organisation, Geneva, $178 \mathrm{pp}$.

Xiao J., Buka S.L., Cannon T.D., Suzuki Y., Viscidi R.P., ToRRey E.F., Yolken R.H. 2009: Serological pattern consistent with infection with type I Toxoplasma gondii in mothers and risk of psychosis among adult offspring. Microbes Infect. 11: 1011-1018.

Yereli K., Balcioglu I.C., Ozbilgin A. 2006: Is Toxoplasma gondii a potential risk for traffic accidents in Turkey? Forensic Sci. Int. 163: 34-37.

Zammit S., Allebeck P., Andreasson S., Lundberg I., Lewis G. 2002: Self reported cannabis use as a risk factor for schizophrenia in Swedish conscripts of 1969: historical cohort study. Bri. Med. J. 325: 1199-1201.

Zhu S. 2009: Psychosis may be associated with toxoplasmosis. Med. Hypotheses 73: 799-801.

Zhu S., Du Y., Li Q., Dong Z. 2007b: High risk of psychosis may be associated with toxoplasmosis. Life Sci. J. 4: 38-41.

Zhu S., Guo M.F., Feng Q.C., Fan J.M., Zhang L-X. 2007a: Epidemiological evidences from China assume that psychiatric-related diseases may be associated with Toxoplasma gondii infection. Neuroendocrinol. Lett. 28: 115-120. 